\title{
AN AIR BEARING ROTATING COIL MAGNETIC MEASUREMENT SYSTEM
}

\author{
Stephen C. Gottschalk ${ }^{\#}$, Kenneth Kangas, David James Taylor, William Thayer (STI, WA)
}

\begin{abstract}
This paper describes a rotating coil magnetic measurement system supported on air bearings. The design is optimized for measurements of 0.1 micron magnetic centerline changes on long, small aperture quadrupoles. Graphite impregnated epoxy resin is used for the coil holder and coil winding forms. Coil holder diameter is $11 \mathrm{~mm}$ with a length between supports of $750 \mathrm{~mm}$. A pair of coils is used to permit quadrupole bucking during centerline measurements. Coil length is $616 \mathrm{~mm}$, inner radius $1.82 \mathrm{~mm}$, outer radius $4.74 \mathrm{~mm}$. The key features of the mechanical system are simplicity; air bearings for accurate, repeatable measurements without needing warm up time and a vibration isolated stand that uses a steel-topped Newport optical table with air suspension. Coil rotation is achieved by a low noise servo motor controlled by a standalone Ethernet servo board running custom servo software. Coil calibration procedures that correct wire placement errors, tests for mechanical resonances, and other system checks will also be discussed.
\end{abstract}

\section{INTRODUCTION}

It is very challenging to measure the magnetic centerline of a quadrupole at the 1 micron level [1]. The measurements are especially difficult when the aperture is small, ca. $13 \mathrm{~mm}$ and the coil is long, ca $600 \mathrm{~mm}$. There are issues related to the coil itself as well as mechanical repeatability and electrical noise. For the coil there is sag under its own weight, size changes due to humidity (especially G10), ease of manufacturing and accuracy of fabrication. In the mechanical system all components need to be extremely repeatable and robust. Finally, there is a practical concern about data acquisition times, electrical signal to noise ratio and means of bucking out a strong quadrupole in order to measure a weaker dipole or sextupole. Our system focused on good centerline repeatability rather than high accuracy.

If high accuracy is needed, but not good centerline repeatability, then a stretched $\mathrm{BeCu}$ wire could be used. The coil sag is orders of magnitude smaller and coil width variations are much less. This is the type of coil that we use for insertion device measurements. The main issues with a stretched wire are the difficulties associated with getting the coil to pass thru a long quadrupole and insuring that the rotation center is very stable. These two requirements imply a mechanically complex design that would be hard to get running with a low probability of success.

In general a measurement system designed to make highly repeatable magnetic centerline measurements must be in a temperature-controlled lab. Ours is controlled to

\footnotetext{
\#scg@stioptronics.com
}

0.2 degC. A lab with $1 \operatorname{deg} C$ temperature fluctuations would give poorer quality measurements. In addition, the temperature changes must be very gradual because of different thermal diffusion times for the materials in the scanner or quadrupole under test. Lastly, the measurement system must be isolated from ground vibrations to prevent excessive shaking of both the measurement apparatus and the quadrupole under test.

We will first describe our rotating coil measurement system that uses air bearings, a graphite coil holder and two coils that can be configured in a bucking configuration [2]. After that we will describe tests that we have performed.

\section{OVERALL DESCRIPTION}

A picture of the hardware is shown in Figure 1. There are two coils connected to a Keithley 2701 Ethernet digital multimeter that is equipped with a 7701 multiplexer card. We connected the coil signals so that the signals routed to the Metrolab PDI5025 integrator would be coil A, Coil B or Coil A+B (bucking). We also connected five thermocouples to the Keithley. The encoder outputs from the Gurley were used to trigger the Metrolab. The particular rotation sequence that we used was to start the coil at -45 degrees and rotate to 405 degrees. The Metrolab was told to start acquiring at 0 degrees. We did not use the Gurley index signal and instead used the servo motor resolver to rotate the coils to a predefined angle. There is a $90 \mathrm{~Hz}$ mechanical resonance on this scanner (discussed later) so we collected 256 points per rotation to avoid aliasing.

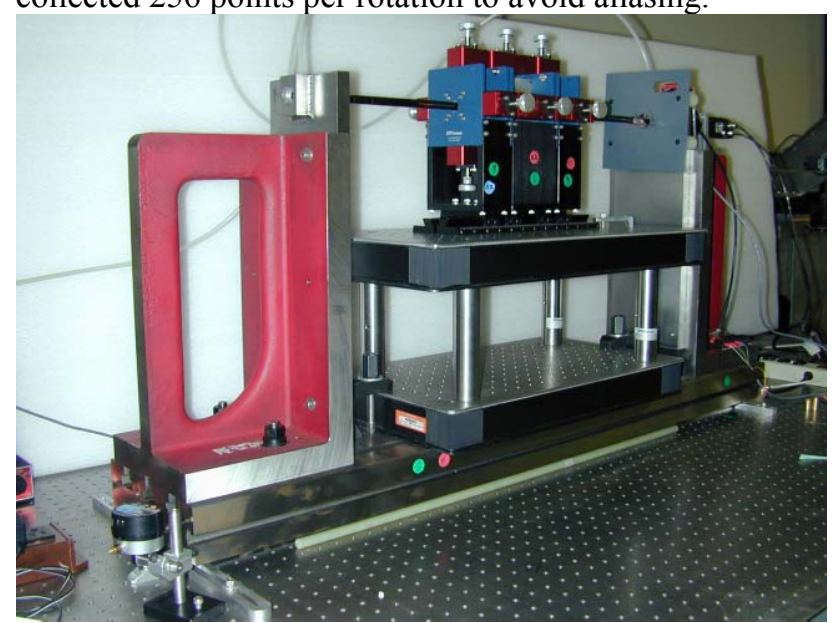

Figure 1: Air Bearing Rotating coil system. A triplet is being measured.

The rotation speed was 1 rotation/sec. Data was transferred from the Metrolab using GPIB and thermocouple readings were transferred using Ethernet. The servomotor was controlled by a Galil DMC2112 
standalone Ethernet motion controller. A low EMI servo drive from Trust Automation was used for the Kollmorgen brushless servomotor. Critical power supplies were linear models, not switchers. A flex coupler connected the coil holder to the servomotor. A Visual Basic program controlled the hardware, collected data, analyzed results and archived compressed scan files onto a network drive that is backed up daily.

\section{AIR BEARING SYSTEM}

We chose air bearings instead of mechanical bearings. The air bearing subsystem has been very reliable and repeatable from the beginning. We had 2 micron centerline repeatability without any temperature control and no vibration isolation within hours of initial setup. No modifications have been made to this subsystem. However, air bearings do require tighter fabrication tolerances than mechanical bearings. The two bearings have to be aligned to a tolerance of $<25$ microns. The ends of the coil holder also need to be equally concentric. If these tolerances are not achieved then the bearing won't rotate.

Figure 1 shows the basic system with a small triplet on the scanner. As can be seen, the system is very simple. It consists of a coil holder that passes thru two air bearings and is clamped to a Gurley rotary encoder. A very small brushless servomotor turns the coil. There is a plastic shield that keeps the coil from snagging on long quadrupoles during rotation. The base is a cast iron Bridgeport bed and the end supports are cast iron ground metrology right angle blocks that have been modified to include air bearing holders.

Air bearings require a clean air supply. We used an automatic draining pre-filter, coalescing filter, desiccant air dryer and sub-micron dust filter. The air pressure was 90 PSI. If we reduced the pressure to 40 PSI then the centerline repeatability was poorer. The system can operate for about 2-3 weeks $24 / 7$ before filters need to be replaced.

The entire system is mounted at the center of a Newport optical table that has pneumatic isolation mounts so the scanner and a quadrupole under test are floating.

\section{COILS}

The particular coil and rod material we chose was a pultruded, continuous carbon fiber (graphite) rod in a vinyl ester base. The ends of the rod were turned down to match the ID of the air bearings. There is a significant mechanical advantage to using graphite for a coil holder. A comparison of rod sag for different materials is shown in Table 1. Since sag scales as the length cubed, this is more critical for longer coils. This rod has $4 \mathrm{X}$ less sag under its own weight than G10. In addition this material has lower thermal expansion and moisture absorption. A final advantage is that this material is not a laminate so it is more dimensionally stable than G10 allowing additional features to be machined into the coil holder.
The potential disadvantage of graphite is its finite conductivity. For a pultruded rod the eddy current paths will enclose very small, random areas. We performed a point-by-point subtraction of the data from a non-bucking coil when the rotation speed was $1 \mathrm{rev} / \mathrm{sec}$ (nominal) and $1 \mathrm{rev} / 10 \mathrm{sec}$ ( $10 \%$ of nominal). The quadrupole had a GL of 67.8T [4]. The only difference was a statistically insignificant centerline shift of 0.3 microns. No quadrupole term could be seen in the analysis. This justified using graphite.

Table 1: Coil holder sag. 3/8" diameter, 24" long

\begin{tabular}{|l|c|}
\hline Material & Sag (microns) \\
\hline G10 & 325 \\
\hline Ceramic (MACOR) & 117 \\
\hline Graphite & 84 \\
\hline
\end{tabular}

There are two coils in the holder that operate individually or in a bucking configuration. This coil geometry is essentially the SLAC rotating coil [1]. We did make a few changes. A drawing of the coil is shown in Figure 2. Notice that no screws are used to hold the coils in the holder. The fit is a light press fit. In addition, there is a central web that is used to increase the stiffness of the rod and locate the coils during assembly. This web cannot be used on G10 rods. The coils are slightly proud of the holder so that they can be held in place by Kapton tape that is wrapped around the outside. After 6 months of operation we have seen no evidence that the coils have shifted inside the holder.

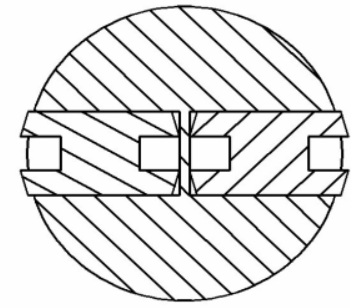

Figure 2: Spinning coil geometry

For these tests we chose to only use 10 turns. The coil forms have room for 50 turns. This does reduce our signal to noise ratio and is one reason why the scanner repeatability is only 0.2 microns. Future coils will use more turns.

The dimensions of both coils were inspected and the widths were found to be within 50 microns of nominal. It is very difficult to balance the sensitivities of such small coils. Our procedure was to define one coil as the reference. Then we adjusted the radial offset of the 2 nd coil until the quadrupole strengths were the same. Finally, we adjusted the angular clocking of the 2 nd coil until the calculated centerlines were in agreement. The final set of coil parameters are summarized in Table 2 .

The 50-micron radial offset between coils caused the quadrupole bucking ratio to be rather poor, only about 70 . This is consistent with the amount of quadrupole directly measured in the bucked coil signal. This simple two coil configuration is not well suited for high accuracy multipole measurements, [2]. On shorter quadrupoles, 
Hall probe scanning [3] is a good alternative. Our experience with the Hall probe method is that it can accurately measure $0.1 \%$ multipoles, but not $0.01 \%$.

Table 2: Coil Parameters

\begin{tabular}{|l|c|c|}
\hline \multicolumn{1}{|c|}{ Parameter } & Reference Coil & 2nd Coil \\
\hline Inner radius & $1.82 \mathrm{~mm}$ & $1.87 \mathrm{~mm}$ \\
\hline Outer radius & $4.74 \mathrm{~mm}$ & $4.79 \mathrm{~mm}$ \\
\hline Angular offset & 0 degs & $-0.29128 \mathrm{degs}$ \\
\hline Number of turns & 10 & 10 \\
\hline Length & $616 \mathrm{~mm}$ & $616 \mathrm{~mm}$ \\
\hline
\end{tabular}

\section{COIL SENSITIVITY VARIATION}

While magnetic centerline measurements do not need a highly accurate coil calibration, for more routine measurements it is good to know how much the coil sensitivity varies with axial position. For these measurements we used one of the short PM quadrupoles shown in Figure 1. The quad strength was 3.8 Tesla, pole length $4.3 \mathrm{~cm}$, aperture diameter $13 \mathrm{~mm}$. This quadrupole was moved to different axial locations and the strength was measured, see Figure 3. The rms variation was $2.15 \%$ which corresponds to a 50 micron coil radius variation with axial position. For the longer center quadrupole in the triplet the variations were less because it is averaging over a length of $8.6 \mathrm{~cm}$.

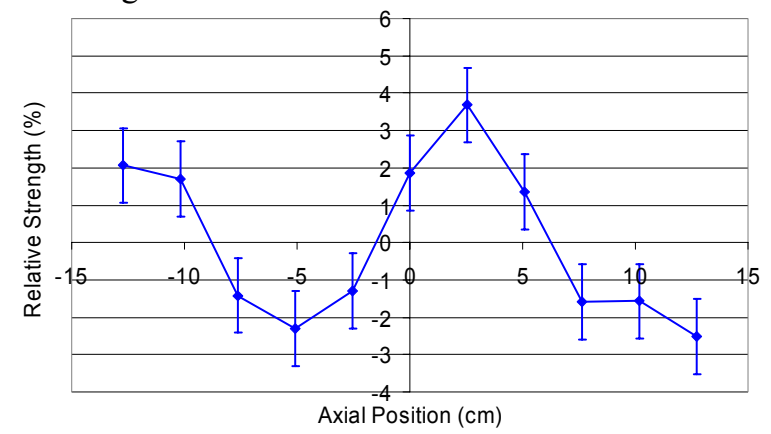

Figure 3: Coil Sensitivity Variation with Axial Position

\section{MECHANICAL RESONANCES}

Since our scanner acquires data at 1 rotation/sec, the dipole (centerline) signal is $1 \mathrm{~Hz}$. High frequency resonances have no impact on the centerline. However, in the future the coil may be spun faster or higher order multipoles may be measured. Then mechanical resonances may be important.

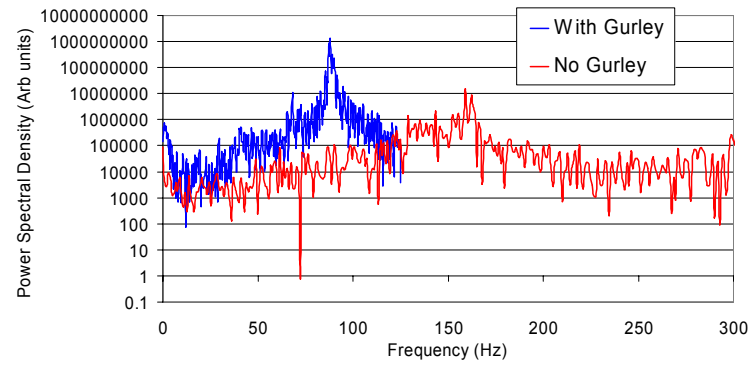

Figure 4: Noise power spectrum measurements.

We made measurements on a stationary coil oriented at 45 degrees in a high field quadrupole [4]. A comparison of the power spectral densities (arbitrary units) with the Gurley attached to the rod and removed is shown in Figure 4. Without the Gurley the resonance occurs at $160 \mathrm{~Hz}$ and has $100 \mathrm{X}$ lower power. Adding the Gurley lowers the frequency to $90 \mathrm{~Hz}$. The resonance shift is caused by the high moment of inertia of the Gurley. This scanner may perform better without it. Further tests are needed to test this hypothesis.

\section{LONG TERM STABILITY TESTS}

We performed long term centerline stability tests on an adjustable strength permanent magnet (PM) quadrupole. This is a test of both the repeatability of this scanner and the stability of the quadrupole. Results for three days of testing are shown in figure 5 . There was a 0.5 micron movement in the centerline that could be either the quadrupole or the scanner. Other tests performed over 12 hrs did not show this movement. We do not know if the scanner or the quadrupole caused it. The short term scan repeatability for this data set was $0.18+-0.08$ microns.

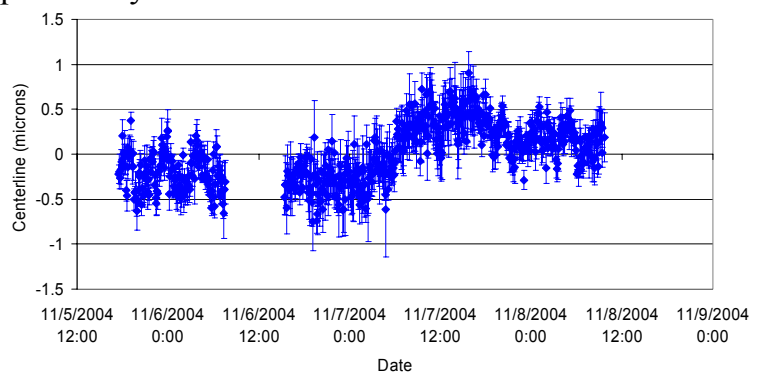

Figure 5 - Long term scanner test results on a PM quadrupole.

\section{CONCLUSION}

An air bearing scanner designed to make highly repeatable magnetic centerline measurements has been built and tested. The air bearing approach is quite attractive due to its ease of use and simplicity. Other coils can be substituted when needed. The spacing between supports can be reduced if required. Short term $(12 \mathrm{hr})$ centerline stability was about 0.2 microns. A graphite coil and holder were tested and there was no evidence of eddy current effects. Mechanical resonances were measured. We found that the primary source of the resonance is a high inertia rotary encoder. Further improvements will be made in the future.

\section{REFERENCES}

[1] C.E. Rago, et al, 'High Reliability Prototype Quadrupole for the Next Linear Collider', IEEE Trans. Appl. Supercond. 12:270-273, 2002

[2] Jain, A.K., 'Harmonic COIL's', Proc. CERN Accel. School on Meas. And Alignment of Accel and De. Magnets, 1997

[3] S. Gottschalk, et al, Nucl. Instr. Meth. Phys. Res. A 507, (2003) 181-185

[4] S. Gottschalk, et al, Particle Accelerator Conference 2005, 'Performance of an Adjustable Strength Permanent Magnet Quadrupole', MPPT029 